By: George F. Michel

Michel, GF. A developmental psychobiological approach to developmental neuropsychology. Developmental Neuropsychology. 2001, 19(1): 11-32.

Made available courtesy of Taylor and Francis: http://www.tandf.co.uk/journals/titles/8756-5641.asp

****Note: Figures may be missing from this format of the document

\begin{abstract}
:
Although both developmental psychobiology and developmental neuropsychology examine the interface between biological and psychological processes, they differ in conceptual framework. This article argues for the incorporation into developmental neuropsychology of certain aspects of the conceptual framework of developmental psychobiology. Three principles of dynamic psychobiological interaction are described and applied to four issues in neuropsychology (handedness, sex differences in behavior, critical periods, and modularity of structure-function relations). Then, it is proposed that developmental psychobiology can make four direct contributions to developmental neuropsychology. Finally, it is argued that the value of the conceptual framework provided by developmental psychobiology depends, in part, on how well it translates into procedures that can be applied in the clinical settings of the developmental neuropsychologist.
\end{abstract}

\title{
Article:
}

The relation between the developing psychological abilities and the developing brain of a growing child has been an object of research and theory for more than 100 years (e.g., Baldwin, 1895; Case, 1992; Gesell, 1946; Gibson \& Peterson, 1991; Johnson,1993; Lenneberg,1967; McGraw,1946; Precht1,1982). The modern discipline of developmental neuropsychology is ideally suited to continue this tradition. Developmental neuropsychology provides both an empirical examination of the relation between the developing nervous system and human psychological functioning and a conceptual framework for characterizing the interface of neural and psychological development. Similarly, the discipline of developmental psychobiology provides empirical evidence and a conceptual framework for comprehending the relation between neural and psychological development as part of its exploration of the developmental dynamics of the biology -psychology interface in the organization of animal behavior. However, because of differences in their historical origins and the types of questions that focus their research activities, these two disciplines differ in the conceptual framework used to acquire and interpret empirical evidence. This article presents an argument for incorporating aspects of the conceptual framework of developmental psychobiology into the framework of developmental neuropsychology.

\section{DEVELOPMENTAL NEUROPSYCHOLOGY}

Developmental neuropsychology arose from the discipline of neuropsychology, which emerged from the combination of two sets of information, one from neurology and one from psychology, neither of which originally incorporated a developmental orientation. In neurology, it is commonly assumed that psychological processes are the consequence of the specific structural organization of the brain (the structure-to-function assumption). Therefore, the neurological information incorporated by neuropsychology consisted primarily of the functional consequences (including social, cognitive, emotional, sensory, or motor disturbances) of various identifiable neural disorders produced by infections, toxic conditions, anoxia, focal damage due to stroke or injury, and such. Of course, most neuropsychologists are mindful that correlating a functional deficit to a particular area of damage only identifies what the damaged brain does to resolve the problem of the missing area. Although the missing area may have supported the function that was disturbed by the damage, it is also possible that the disturbance is the consequence of the functional reorganization after damage. Notice that this rather small semantic difference represents a major theoretical difference when attempting to construct an explanation of brain -behavior relations. 
However, within the pragmatic constraints of focusing on clinical populations, the collection of information about the relations between psychological function and neural dysfunction is intended to provide a precise description of symptoms associated with disruption of specific brain areas. This information permits assignment of a patient to a syndrome category on the basis of the symptoms. These syndrome categories permit clinicians to predict the expression of potential symptoms based on the specific brain areas affected by the neurological condition. Conversely, the pattern of symptoms expressed by the patient are used to predict which specific brain areas are likely to be affected. Obviously, the success of such prediction improves with the quality of the assessment techniques that are employed. Therefore, the discipline of neuropsychology incorporates psychology's most sophisticated assessment techniques. To aid the neurologist in the assessment of the correlation of functional deficits with neural disorders, the neuropsychologist often administers to patients a battery of widely accepted and approved standardized tests of general and specific psychological functioning. When the patients are children, standardized tests with specified "age-norms" are often employed so as to chart a patient's relation to a population of similarly aged individuals.

Obviously, psychological functioning is a complicated phenomenon. To understand it, it must be segmented into smaller chunks for examination and analysis. Modern psychological assessment techniques segment psychological functioning into a large number of faculties. A major concern in psychology is whether the categories of psychological functioning assessed by the tests are meaningful units or merely arbitrary divisions. Therefore, when the pattern of functional deficits is associated with specific neurological disorders, this correlation seems to provide a "natural cleavage" for the identification of fundamental categories of psychological phenomena.

For example, neuropsychological case studies of brain damaged individuals support divisions such as between

1. Certain aspects of speech production and certain aspects of speech comprehension.

2. Specific visual-spatial tasks and certain linguistic functions.

3. Lexical and nonlexical process of reading aloud.

4. Surface and deep dyslexia.

5. Implicit and explicit memory.

Thus, neuropsychological data seem to provide the identification of "natural" categories of psychological phenomena. In this manner, neuropsychology provides support for the validity of specific assessment techniques that are thought to identify natural categories of psychological functioning.

The techniques employed by neuropsychologists, when unencumbered by conceptual confusions and methodological difficulties, permit neuropsychological research and practice to take the form of "normal science" (Kuhn, 1962). That is, the application of well-understood "standard" techniques (e.g., standard psychological testing instruments) to well-defined problems (individuals suffering various sorts of brain damage) provides conventionally acceptable interpretations of brain-psychology relations. However, neuropsychology extends beyond "normal science" when neuropsychological procedures are applied to individuals who are not suffering from conventional neural disorders. This extension appears to be justified by a theoretical explanation of brain -behavior relations in which it is proposed that a specific neural process is responsible for a specific score on a psychological test.

Although the evidence of neuropsychology reveals that a known neurological disorder is associated with an observed pattern of psychological test scores, this does not mean that any similarity of the pattern of psychological scores of otherwise healthy individuals implies a similar, albeit "milder," neural disorder. The common occurrence of this mistake is revealed when neuropsychologists use patterns ofperformance on their psychological assessment tests to propose notions of "soft neurological signs" or "minimal brain dysfunction" for individuals not known to have a neurological disorder. Reasoning from psychological test scores to neural 
processes can only be justified if neuropsychologists assume that their correlation procedures can provide evidence for explanatory theories for brain -behavior relations.

Of course, assessment of the validity of any theory requires examination of the conceptual framework within which the normal activities of the discipline are conducted. Such examination usually includes the critical investigation of conventionally accepted and familiar techniques, concepts, and assumptions to identify the conditions on which their justification and usefulness depend. Such criticism does not imply acceptance of a brain -behavior dualism. Applying such critical investigation to neuropsychology means questioning the meaning of standardized test results, the structure-to-function assumption of brain -behavior relations, and the value of case studies. This disrupts the normal routines of the discipline and too often leads some to dismiss these endeavors, and the debates that ensue, as mere semantics.

Of course, neuropsychologists frequently debate the proper methods that should be used in constructing theories of brain -behavior relations and they readily acknowledge the complexity of the dynamics of the processes generating these relations. However, too often the observed correlation between brain damage and alterations in psychological functioning is used as evidence in support of one sort of hypothesis about brain-behavior relations and for the disconfirmation of other hypotheses. Thus, case studies are accorded the status of experimental manipulations (Shallice, 1988). Then these "supported" hypotheses are used to explain normal brain -behavior relations or to design research on individuals not suffering from brain disorders. Although this approach in neuropsychology has been criticized frequently (e.g., Efron, 1990; Ellis, 1987; Marshall, 1980), it is especially misleading in developmental neuropsychological research.

Psychological functions develop through the interplay of various factors that are internal and external to the boundaries of the individual. Although it is quite fashionable to acknowledge such interaction in development, too often it fails to affect either research designs or the interpretation of results. Within the conceptual framework of developmental psychobiology, it is directly acknowledged that the complex patterns of interaction between social and environmental experiences and neurobiological growth processes may not be revealed by conventional research designs. Neurobiological processes participate in a continual dynamic interplay with social and environmental experiences in which feedback, reciprocal influence, and circular causation occur. Consequently, neuropsychological development will exhibit nonlinear causality. To understand such development, it is necessary not only to identify which factors play a role, but also to identify the processes by which they exert their influence. Analyses of the end-product of development, its achievement (e.g., a test score), would not allow accurate reconstruction of the processes involved in generating that product. Moreover, standard techniques and conventional concepts that may be suspect in adult neuropsychology are even more questionable in developmental neuropsychology. New principles, concepts, and techniques must be employed. The discipline of developmental psychobiology may be a good source for some of these new principles, concepts, and techniques.

\section{DEVELOPMENTAL PSYCHOBIOLOGY}

Much of the theoretical and empirical work on the dynamics of the biology-psychology interface that has occurred within the discipline of developmental psychobiology may be unfamiliar to many psychologists and biologists. This unfamiliarity stems, in part, from a common tradition of defining psychobiology primarily as any research activity that simply combines biological techniques with psychological topics. Hence, any activity in which a conventional psychological topic (e.g., learning, motivation, memory, perception, attention, aggression, sleep, or psychopathology) is examined in relation to a conventional biological technique or procedure (e.g., measures of neural physiology, neurochemistry, or anatomy) becomes psychobiology. However, several theorists have proposed that the meaning of psychobiology in developmental psychobiology implies integration of biology and psychology at a more fundamental level (cf. Michel \& Moore, 1995).

Developmental psychobiology was derived in part from a natural history approach to the study of animal behavior. That approach required adoption of a multileveled (from ecosystem to cellular processes) examination of an organism. Also, the organism is expected to have an adaptive relation to its environment throughout its 
lifespan. This results in a somewhat unconventional view of development (see Gottlieb, 1992). One aspect of that view is that natural selection has influenced how development should take place within the typical environment of each species. Thus, the development of a behavior pattern may appear extremely "innate" and constant in all or nearly all individuals of a species because natural selection has assured the compatibility of the interaction of the "species-typical" environment and the individual's genetic-developmental processes. Social and environmental experiences will provide subtle contributions to both developmental stability and change throughout the life span. The only way of discovering these environmental contributions is to systematically manipulate relatively ubiquitous features of the environment. Therefore, alterations in seemingly minor aspects of the environment may produce quite different development; whereas, alterations in seemingly major aspects may not alter development at all.

The same genes, in an environment different from that in which evolution took place, can produce individuals with quite different characteristics. This is especially relevant for understanding the development of both domesticated animals and humans because they exist in an environment quite different from that in which natural selection originally operated. This means that even if natural selection favored individuals with some trait (e.g., aggression), they are not irrevocably committed to being so. Developmental psychobiological investigations can reveal which environmental conditions might be manipulated to alter the development of any particular characteristic. Also, within this framework, the discovery of some unusual or anomalous characteristics of individuals (similar to case studies in neuropsychology) does not permit evaluation of specific hypotheses about the character of the psychology -biology interface. Rather, such cases raise questions about development that prompt experimental investigation. Such investigation often reveals complex patterns of interaction between experience and neurobiological processes during development that were unsuspected at the time of the original discovery (e.g., imprinting, bird-song, hoarding food, recognizing predators, recognizing food, using cues for migratory navigation, etc.; cf. Blass, 1986, 1988, and Hogan \& Bolhuis, 1994).

As a result of their different ancestry, developmental psychobiology and developmental neuropsychology characterize the biology - psychology interface rather differently. Therefore, they will differ both in the manner by which research is designed and conducted and how results are interpreted. Moreover, because developmental neuropsychologists are motivated, in part, by clinical issues, and developmental psychobiologists are motivated, in part, by the acquisition of knowledge for its own sake, they will differ in the type of results that are considered pertinent. The immediacy of patient needs will shape the theoretical program of neuropsychology. Nevertheless, it is my contention that some of the ways by which developmental psychobiology approaches the study of brain -behavior relations ought to be incorporated into the framework of developmental neuropsychology.

In this article, I describe three principles of developmental psychobiology concerning the dynamics of development that relate to the biology -psychology interface. Then, I briefly examine four issues in developmental neuropsychology (handedness, sex differences in behavior, critical periods, and modularity of brain structure and function) from a developmental psychobiological perspective and in relation to those three principles. Finally, I identify four contributions that developmental psychobiology can make to the conceptual framework of developmental neuropsychology. My hope is that the particular twist on these notions from developmental psychobiology will be useful as developmental neuropsychologists approach their task.

\section{DEVELOPMENTAL PRINCIPLES}

\section{Developmental Explanations Involve a Multiplicity of Causal Factors}

One principle to be derived from the notion of dynamic interaction is that the identification of one factor as contributing to the development of some characteristic does not preclude conclusions about the importance of other factors. For example, discovery of the contribution of a genetic factor should not preclude discovery of the importance of nongenetic factors, including experience. Conversely, identification of an experiential contribution to development of some psychological function should not preclude discovery of a genetic contribution. Moreover, both genetic and experiential constructs require "unpacking" by developmental investigations that identify intrinsic and extrinsic genetic components and, in mammals, maternal influences and 
self-generated social and environmental experiences hidden in behavior-genetic constructs (Atchley \& Hall, 1991; Michel \& Moore, 1995). In unraveling the dynamic interaction in development it becomes apparent that all factors that have been discovered to contribute to the development of some function are equally important and that additional factors may yet be discovered. Consequently, if one type of experiential factor is demonstrated as not influencing development, this does not imply that other experiential factors are equally unimportant. The developmental psychobiological study of handedness and sex differences in reproductive behavior provides good examples of the value of this principle.

\section{Developmental Explanations Require Specification of How Development is Accomplished}

A second principle derivable from a dynamic interaction is that the identification of the factors affecting development does not reveal how they accomplish their effect. A study which identifies that a difference in some factor (e.g., size of some brain structure or type of experience) relates to a difference in developmental outcome does not reveal the process by which that difference produced the change. Developmental psychobiological research must describe exactly "how" that factor affects development and often such investigation reveals important, but not obvious, contributions of individual experience. The developmental psychobiological study of sex differences in adult behavior identifies exactly how perinatal hormones contribute behavioral differences in adulthood.

\section{Developmental Explanations Require the Study of Development}

Too often, the discovery that some ability or function is present early in life (e.g., at birth), and is similar to that of the adult, ends the developmental investigation. Or, if some ability appears to develop independently of typical variation in social or physical environmental conditions, this may bring investigations to a close. Instead, such results should provoke additional attempts to comprehend the development of the ability. The origin of the ability and the developmental consequences of the ability must be identified.

Identifying aspects of the individual that do not respond to typical variations in environmental conditions only specifies a problem for developmental investigation; it does not provide an answer about how the individual's ability develops. Also, the elicitation of adult-like patterns from immature individuals need not indicate presence of adult neurobehavioral organization. Although elements of the neurobehavioral system that typically enter into the organization of the adult pattern may be present earlier, these early occurring elements may depend on the presence of environmental support conditions that are unnecessary for the adult. Empirically derived explanations that account for the differences in sensitivity to contextual support between early and adult abilities must become part of the developmental account of the adult pattern.

\section{FOUR ISSUES IN DEVELOPMENTAL NEUROPSYCHOLOGY}

\section{Handedness}

Hemispheric specialization of function is one of the most intriguing and popular issues in neuropsychology. Various kinds of research on clinical and nonclinical populations indicates that the organization and control of speech and the comprehension of both written and auditory language is unevenly supported (i.e., lateralized) by neural mechanisms in the right and left hemispheres of the brain. Certain spatial abilities are also unevenly supported by the two hemispheres but in apparent opposition to the lateralization of language abilities. It has been proposed that the neural mechanisms of the left hemisphere are better able to support manifestation of language-like abilities, whereas the neural mechanisms in the right hemisphere are better able to support manifestation of certain spatial abilities.

It is interesting to note that most researchers choose right-handed males, without a family history of sinistrality, to investigate hemispheric specialization of function (Bryden \& Steenhuis, 1991). Conventional wisdom, supported by the results of some studies, is that the mechanisms supporting left hemisphere language abilities are present in nearly all right-handed individuals but maybe less so in left-handed individuals. Also, males are considered to be more strongly lateralized than females and those with familial sinistrality are considered to be genetically predisposed to sinistrality and hence the character of their hemispheric specialization is more problematic. Of course, these notions reveal the importance of understanding handedness for understanding 
lateralization and hemispheric specialization of function. However, they also raise the question of whether hemispheric specialization is of much adaptive significance for humans because right-handed males without a family history of sinistrality represent only a minority of the population.

Because handedness is both an aspect of hemispheric specialization and an influence on the lateralization of other functions, it is a fundamental issue in neuropsychology (Bryden \& Steenhuis, 199 1). Yet, its development has been relatively ignored. Perhaps, it is assumed that handedness reflects the development of neural mechanisms underlying lateralization that are under genetic control. Indeed, various genetic models can predict the pattern of expression of hand-use preferences in a population (Annett, 1995; Corballis, 1995; McManus \& Bryden, 1992). However, genetic models must be unpacked by developmental investigation to reveal how the characteristic is acquired (Principle 1). My own work on the development of handedness (Michel, 1987, 1998) demonstrated that when unpacked by developmental analyses, many intrauterine and postnatal experiences are shown to play important roles in development of an individual's handedness.

There is a relation between the specific direction of neonatal supine head orientation preference, as comprised by a general postural asymmetry, and subsequent hand-use preferences in infants (Michel, 1981). Using systematic longitudinal observations and simple manipulations, we were able to deduce that the relation is derived from self-generated experiences (Principle 2). That is, head orientation preference, as a component of lateral asymmetry of neonatal posture, results in lateral asymmetry of visual regard of the hands and an asymmetry of hand and arm actions (Michel \& Harkins, 1986). The hand and arm actions are not components of the postural asymmetry but rather are induced by the direction of head orientation. Similar asymmetries of visual regard and limb action in animal models can result in asymmetries in the functional architecture of the various structures of the nervous system associated with the use of the limbs (Spinelli \& Jensen, 1982). We proposed that neonatal postural asymmetries contribute to the development of hand-use preferences (Principle 2) via their influence on the development of asymmetrical neural architecture supporting hand and arm actions. This asymmetry, in turn, contributes to the development of lateralized asymmetries in the ability to coordinate visually guided object prehension and manipulation. It is likely that such changes in asymmetrical architecture can be accomplished by alterations in denditric growth in various cortical and subcortical structures as a consequence of coincidental activity of neurons (Quartz \& Sejnowski, 1998).

Although the neonatal postural asymmetries have an impact on neonatal left and right hand fisting patterns and arm movements, as well as visual regard of the two hands, neither the neonate's posture nor the hand asymmetries were simply early expressions of the same neural mechanisms responsible for handedness (Principle 3). Rather, systematic investigation of hand-use at different phases of development revealed differences in the range of conditions under which a hand-use preference may be manifested, thereby identifying differences in its organization (Michel, 1998). The reaching preference of a 6-month-old infant is not the same as that of a 12-month-old, much less the same as the handedness of a 6-year-old child.

Our research revealed, also, that the specific strength of an infant's preference is affected by the handedness of the mother. The mother's effect is manifested in patterns of toy-play with the infant (Michel, 1992). Thus, maternal handedness can influence the direction of an infant's hand-use preference only for those infants with initial very weak preferences. Otherwise, individual handedness seems to develop from a neonatal manifestation of a head orientation preference prompted by a postural asymmetry that may reflect intrauterine influences (Previc, 199 1). Thus, the majority of infants exhibit a rightward head orientation preference that promotes laterally asymmetric sensorimotor experiences, which contributes to an early right hand-use preference. A leftward neonatal head orientation preference is associated with an early left hand-use preference. An initial preference in prehension facilitates the exploration of objects and the elaboration of manipulation skills that yields a later manifestation of unimanual manipulation preference. Manual differences in manipulation skill yield subsequent differences between hands in "bimanual" manipulation actions that result in bimanual handuse preferences. Subsequent hand-use continues to build on these early preferences resulting in the expanded domain of preference associated with adult handedness. If handedness, a form of lateralization that affects other forms of hemispheric specialization, develops in this manner, might not other lateralized functions? Thus, a 
developmental psychobiological approach to the study of handedness can provide a different perspective from which to approach the development of hemispheric specialization of function.

\section{Sex Differences in Behavior}

The conventional notion is that men and women differ in many aspects of brain -behavior relations. There is extensive documentation of sex differences in psychological functioning. Sometimes, these differences are considered to reflect differences in child-rearing and socialization. However, many sex differences in psychological functions do not vary across cultures, and they do not appear to be deliberately taught or fostered in childrearing. When research reported the neuroendocrine mechanisms underlying sex differences in the reproductive behaviors in rats, similar mechanisms were proposed to operate in humans and the rat became a model for theoretical explanations of species-typical human sex differences and even of sex partner preferences (LeVay, 1991).

Although most of the sex differences in the behavior of rats are dependent on specific sex typical hormones associated with puberty, many of these differences are not reversed simply by switching the presence of these hormones in adults. The nervous system seems to be sex differentiated in its sensitivity to sex typical hormones. The sex differentiated component of the neural sensitivity to pubertal hormones appears to be dependent on prenatal and neonatal exposure to certain hormones typically associated with the male genotype (Yahr, 1988). Alteration of the rat pup's early hormonal condition could reverse sex-typical sensitivity to pubertal hormones and reverse sex differences in adult behavior. In consequence, it is generally assumed that the mammalian nervous system differentiates as female unless it is exposed early in development to certain hormones (e.g., testosterone) associated with the male genotype. Early exposure to testosterone, which typically occurs to genetic male mammals, leads to the development of neural architecture that supports the manifestation of maletypical behaviors.

However, the systematic research by Celia L. Moore (cf. 1992, 1995) has challenged this model and provided a dynamical interaction model of the development of sex differences in behavior for rats that appears to be even more relevant for understanding human sex differences. Moore observed that each mother rat treats each of her male offspring differently than each of her female offspring in a very specific way. The different treatment is primarily in the time the mother spends licking the anogenital area of her pups. Mother rats must engage in anogenital licking to enable their offspring to urinate and defecate. Otherwise, the young would die before they become competent at these activities. During anogenital licking, the mother also recycles many of the nutrients and most of the water lost through nursing the young. Although all pups receive anogenital licking, males receive far more of it than do females. During this licking, male pups also exhibit greater sensitivity of maternally elicited "reflexes" that facilitate further licking by the mother.

Moore (1995) found that the perineal odor, associated with the male pup's urine, attracted the extra attention from the mother. The perineal odor is a consequence of the early secretion of testosterone in the male and may involve its impact on the development of the preputial gland, as well as the production of metabolites in the urine. When Moore made mother rats insensitive to odor of the urine, they could not distinguish males from females and these male offspring were then reproductively and behaviorally deficient as adults. Moreover, if female pups are provided with additional anogenital stimulation either artificially or by making that area attractive to mothers, then, as adults these females will exhibit more male-like behavior. This is especially significant because these females were not exposed to early testosterone; yet their nervous system, like that of a typical male, is more sensitive to male-typical pubertal hormones. Moreover, although the male rats that were not treated as males by their mothers had the same exposure to neonatal testosterone levels, as adults, they were deficient in their sensitivity to male-typical hormones, as are normal females.

Other research (Moore, Dou, \& Juraska, 1992) showed that anogenital licking has an impact on the size and architecture of the sex differentiated neural structures involved in the manifestation of sex differentiated behavior (Principle 2). Thus, the development of sex differences in behavior of rats represents a dynamical interaction among neonatal and adult hormones, neonatal reflexes and odors, and maternal behavior; none one 
of which is more important than the other (Principle 1). The anatomical and neural architectural structures supporting sex-differentiated behaviors are a consequence of a complex pattern of hormonal and experiential influences extended over time. Although some of these differences in experience are prompted by sex differences in certain behavioral "reflexes" of neonatal pups that exhibit a similarity to adult behavioral differences, Moore (1995) found that neonatal reflexes differed from that of the adult in both pattern and contextual support (Principle 3).

Generalizing from Moore's (1995) research, we might expect that subtle but powerful experiential influences contribute to the development of species-typical sex differences in humans. Rather than being satisfied with a demonstration that some differences in psychological functioning between males and females occur crossculturally or relate to sex differences in gross neuroanatomy, developmental neuropsychologists should be seeking to identify the developmental origins of such differences. Even when there is no obvious attempt to promote sex differences during childrearing or when sex differences occur early in the life of the child, it is possible that subtle experiential influences are contributing to their development.

\section{Critical Periods}

The concept of "critical period" is used to describe the notion that at certain points in development the individual is more receptive or vulnerable to environmental influences than at other points. One text in developmental neuropsychology uses the criteria specified in Nash (1978) for identifying a critical period (Spreen, Risser, \& Edgell,1995, p.140). Supposedly, these criteria indicate that the sensitivity of the individual to an external stimulus must be triggered by some internal (maturational) fact or with specific onset and "offset" ages. The condition of a specific onset and offset age, timed by endogenous factors, is crucial to the use of critical period in the developmental process. Crucial, also, is the notion that development of some ability is no longer open to experiential influences once offset has occurred. Thus, neuropsychological data demonstrate that both language development and second language development have a critical period during which exposure to the language must occur, otherwise adult language ability is deficient (Gitterman, 1999).

Too often definitions of critical periods are not distinct from the definition of development. For example, Colombo (1992) defined critical period as

The time between the emergence, anatomically or functionally, of a given biobehavioral system and its maturation. The system maybe affected (for better or for worse) in this emergent, but immature state, by exogenous stimuli and this effect can be permanent should the system 'harden' to maturity. (p. 263)

However, does not the development of any ability usually refer to the time between its initial emergence and the achievement of its "adult" or constant form? If so, then according to Colombo, all of development is a critical period.

The concept of critical period derives from 19th century embryology, when it was discovered that developmental trajectories of certain anatomical structures could be more easily altered by environmental manipulations at certain stages than at others. When employed in the study of teratogenic agents in embryology, the effect of the teratogen (an external stimulus, usually chemical) on subsequent development was maximal only at certain stages and the critical stage differed for different structures. However, when the concept was incorporated into natural history studies of animal behavior and subjected to rigorous developmental psychobiological examination, the concept lost much of its explanatory power (Bateson, 1981). Consequently, the concept was changed to "sensitive period," to describe certain developmental phenomena.

Sensitive period refers to the observation that the development of some specific behavioral characteristic of the animal is especially sensitive to the influence of certain stimuli, typically available in the natural environment, at a particular stage of its development. However, that same behavioral characteristic can be influenced by other, nontypical, stimuli at other stages of development (Principle 1). Moreover, both the onset and offset of the sensitive period is influenced not simply by internal processes but by previous experiences (Principle 2), 
particularly self-generated experiences (cf. Michel \& Moore, 1995, pp. 38-43, 407-409). Thus, in the concept of sensitive period, the notions of onset and offset "age" are replaced by empirically derived knowledge about the relation between the stimulus conditions and the developmental status of the individual, and that status is only minimally related to age (Bateson, 1979). Such knowledge permits greater flexibility for both the onset and offset of the period. The specific timing of an experiential event is less important than that the experiential event relate to the specific status of the individual. The concept of sensitive period preserves the sequential character of developmental processes without requiring adherence to a specific temporal order for development. Moreover, it does not close inquiry into how typical and nontypical experiential events contribute to both further stability and change in development.

Although the concept of critical period may provide some descriptive service in the investigation of unusual conditions that disrupt normal development, it is too misleading to use for understanding normal development. In contrast, the concept of sensitive period preserves the descriptive function but alerts the user to its limits as an explanation of development. Moreover, it prompts the user to continue the search for conditions that might promote change even in the hardened, mature system. From a developmental psychobiological perspective, the concept of critical period need not be incorporated into the conceptual framework of developmental neuropsychology.

\section{Modularity of Brain Structure and Function}

Until recently, most models of cognition assumed a centralized, linearly structured hierarchical organization with an executive control process. Executive processes or routines were presumed to coordinate perception and action. A similar organization was presumed for brain structure-function relations. Researchers sought specific areas of the brain that presumably created and controlled the programs for coordinated action, complex perceptual abilities, comprehension and production of speech, reading and writing, and so forth. However, the evidence that normal individuals can perform simultaneously two complex demanding tasks without detriment to either and that neurological patients can exhibit syndromes in which they have deficits in specific grammatical rules but not others, and so forth, has led to the adoption of a modular notion of brain -behavior relations. That is, it is presumed that cognition is composed of many semi-independent and separate, but interconnected, processors and the brain's structure represents this modular architecture. The latter is consistent with modern neurobiological accounts of cortical organization in which certain functions appear to be related to specific anatomical columns (De Yeo, Felleman, Van Essen, \& McClendon, 1994; Merigan, 1993).

The modularization of cognitive functions automatically lends itself to a diagrammatic representation. The functions can be arranged within a diagram that both separates them and identifies their interconnections. Although this is not necessary, these diagrams often presume a modular architecture that permits some components to perform normally when others may have been totally eliminated. Thus, the consequences of damage to a module or a connection can then be determined and predictions made about the deficits in cognitive functioning. Modern neuropsychology employs a model of brain -behavior relations that assumes individual components function in the same manner, whether they are joined together or disconnected. Indeed, current neuropsychological theory depends on the presumption of distinct cognitive modules that are also spatially separated in the brain (Bradshaw \& Mattingley, 1995; Shallice, 1988).

There can be little argument with the fact of modularity, only about its nature and extent. The contents of any module mustbe unpacked and the details about how it operates must be determined. Finally, for developmental neuropsychologists, the developmental history of the module must be identified. For example, only a small minority of humans have ever achieved the ability to read and write with alphabetic script. However, literacy appears to establish cognitive modules that can be selectively impaired by brain injury to produce a wide variety of different forms of reading and writing disorders. Although some may argue that literacy builds on "hard-wired" language modules, or certain combinations of language modules and visuo-spatial modules, there are reports of individuals with brain damage who show impairment of reading and writing skill without apparent impairment of other cognitive functions. Of course, from the perspective of developmental 
psychobiology, the modules must be constructed during development and the notion of hard-wired modules is misleading.

There is evidence from developmental neurobiological research that the apparent structural modularity of the brain arises as an epiphenomenon of simple developmental growth processes (Purves, Riddle, \& LaMantia, 1992, 1993). Moreover, experiential processes profoundly affect neocortical structure-function relations, even in adults (Darian-Smith \& Gilbert, 1994; Merzenich, Recanzone, Jenkins, \& Nudo, 1990). Developmental psychobiological (e.g., Bekoff, 1988) research demonstrates that functions that are modular at one level of description (e.g., behavioral) are intermingled at another level (neural). Therefore, the observation that an individual can perform simultaneously two demanding tasks does not imply that they are subserved by distinct and separate sets of neural modules.

The characteristics of dynamic systems theory (Michel, 1991; Thelen \& Smith, 1994) appear to allow separate functions to be created from a multiplicity of interdependent processors. That is, within the context of ongoing cycles of neural activity, signal enhancing processes emerge in relation to task, biomechanical, and contextual constraints. There is no executive control hierarchy but rather a coalitional heterarchy in which information and control activities are decentralized and interactive. Within the constraints of task, biomechanics and context, the ongoing neural activity either achieves a state of dynamic equilibrium or not. If not, some level of equilibrium must emerge eventually; otherwise, the organization disintegrates. When a perturbation (alteration in task or context) challenges a state of equilibrium by exceeding the buffering capacities of that state, a series of fluctuations begin. From within the context of this systemic disorderand disequilibrium, a new dynamic equilibrium is afforded. This resultant equilibrium is not a "set point" but a dynamic state. Although it remains to be determined whether dynamic systems theory can provide the basis for comprehending neuropsychological phenomena without the postulation of hard-wired modules of structure-function, it is consistent with a developmental psychobiological perspective.

\section{CONTRIBUTIONS}

There are four contributions that a developmental psychobiological approach can make to the field of developmental neuropsychology. The first contribution is that during development, new functional achievements derive from abilities present at earlier phases. However, developmental psychobiological investigations have revealed that the derivation may not be constrained by any functional categorization of the abilities or by our perception of such categorization (Fentress, 1991). That is, abilities that are apparently functionally distinct and separate may be developmentally related. This developmental relation is identifiable only by empirical investigation. For example, infant supine kicking activities may be related to both neonatal "reflexive stepping" patterns and subsequent initial walking patterns, although both intuitively and perceptually, kicking appears to be a functionally distinct pattern (Thelen \& Cooke, 1987). It is likely, therefore, that supine kicking activity may play an important role in the development of bipedal locomotion. Similar developmental psychobiological investigations of prehatching spontaneous leg movements of chickens revealed their developmental relation to postnatal locomotion abilities, despite apparent differences in pattern (Bekoff, 1988). Thus, developmental causality may not be identified necessarily by either an immediate temporal relation or by an obvious functional similarity among phenomena.

For the developmental neuropsychologist, the development of initial reading ability may not require practice with, or even exposure to, books or other reading materials; rather it may depend on the development of certain forms of neural processing derived from motor skills acquired some time before reading begins. Some difficulties in the acquisition of reading abilities may reflect differences in the timing of cognitive processes that, in turn, may have derived form mechanisms involved with motor control (Wolff, Michel, Ovrut, \& Drake, 1990). If so, then reading acquisition may be facilitated in individuals with dyslexia by creating alternative procedures for timing the cognitive processes involved in reading. Perhaps, the success associated with individual tutoring programs for dyslexia resides in the inadvertent discovery of such alternative procedures. 
Typically, the developmental neuropsychologist will start a developmental inquiry by focusing on the "finished" adult product and then search for earlier elements of this product. Because development likely builds on the available raw materials, there is no logical reason that recycling of earlier achievements should be restricted to functionally similar categories. Hence, developmental psychobiological research typically begins with the abilities of the "younger" individual and that individual's commerce with the sociocultural and ecological context to identify where these abilities lead (Michel \& Moore, 1995). In this way, precursors of adult abilities can be found which initially may not have been intuitively obvious. This does not presume that the individual begins as a tabula rasa. Rather, it presumes that the structure of the initial system may not be inherently the same as the adult structure.

The second contribution is that the development of the nervous system depends on its context. That context can include

1. Hormonal conditions (that, in turn, may be dependent on sensory stimulated neural events).

2. Biomechanically created patterns of sensory feedback prompted by spontaneous activity of motor processes.

3. Behaviorally created alterations in the social and physical environment that affect sensory systems which, in turn, alter hormonal condition and neural state.

Hence, brain and behavioral development are in reciprocal interdependence. Whenever the development of a psychological ability is demonstrated to depend on the developmental achievement of a specific neural state, the development of that neural state is likely to have depended on the achievement of a specific social or physical environmental condition prompted by a previous behavioral event.

This means that neuropsychological development will exhibit a coactional nonlinear causality that will require investigations to begin with the specification of abilities and states at one phase and then to try to identify their consequences for subsequent phases of development (development from). Also, this means that the individual's development will be influenced by self-generated experiences. That is, the social and physical environmental consequences of the individual's own actions will generate sensory experiences that will contribute toward the individual's subsequent neural development. Also, neuropsychologists will have to expand their expertise to include hormonal, immunological, biomechanical, and other nonneural factors when seeking the causes of behavior.

The third contribution is that the individual is both a structured system and a part of a structured system (an Umwelt of perceivable social and physical environmental events). This means that different combinations of internal and external conditions and events can form similar achievements and that quite different achievements can emerge from rather slight variations in these combinations. Hence, developmental "timing" (e.g., critical periods, "readiness" phenomena, and "sleeper" effects) depends on combinations of internal and external events, not on internal "clocks." Although certain neural structures exhibit clock-like characteristics, and these may be incorporated into simple rhythmic events, developmental phenomena are not likely to be fully explained by such structures. The timing and sequential organization of developmental phenomena are not the consequence of internal control systems. This means that "vulnerability" will fluctuate because the effects of the same perturbation will be minimal or maximal depending on the "state of coherence" of the individual-context system at the time of the perturbation. Thus, to understand how a perturbation can have an effect will require understanding the state of coherence of the individual in context.

The fourth contribution is that developmental neuropsychology has greatly benefited from incorporation of research conducted on animal models of human phenomena. However, adoption of a developmental psychobiological perspective will allow greater incorporation of natural history oriented animal research. A natural history orientation to the study of animal behavior means that the research is

conducted to comprehend the animal's behavior in its natural setting (Lehrman, 1971). The emphasis is on understanding the animal for its own sake and not as a model of human phenomena. Humans are extensively 
symbolic in so many aspects of functioning that animal models can match the realms of human neuropsychology only imperfectly. Animals can only be weak models of human neuropsychological functioning, although they can reveal basic processes by which neural architecture may be organized and reorganized by function.

The investigation of the biopsychology of various animal species can provide a conceptual context within which the conceptual framework by which we examine human biopsychology and neuropsychology can be challenged (e.g., Rovee-Collier, 1996). That is, in attempting to examine ourselves, we must be able to generate a frame of reference that is not completely dependent on the original object of examination (i.e., ourselves). Therefore, if we are to avoid simplistic confirmation of conventional wisdom and potential self-delusion, we must explore the development of other species so as to challenge and break our anthropocentric frame of reference. Of course, it is not possible to completely eliminate the anthropocentric bias in the study of other animals. However, when we approach that study with the intent to discover the animal's "world" rather than our own, we can achieve a perspective on both the animal's world and our own that is less based on our socially and culturally derived self-reflective intuition (Dethier, 1969). That is, the animal will challenge our attempts to cast it as a simplified form of human. From this comparative perspective, we can come to better understand ourselves.

\section{CONCLUSION}

One impediment to understanding brain-behavior relations is that it incorporates an issue that affects theory choice in science in general - the issue of parsimony. The assumption of parsimony means that we will prefer the simplest conceptualizations of brain -behavior relations. Our preference for intuitively appealing explanations of any complex phenomenon might lead them to appear to be simpler and hence, intuitively appealing explanations for brain -behavior relations will appear simpler and more parsimonious. Lay physics is simpler for most people than scientific physics. Folk psychology is simpler than scientific psychology. Are explanations that appear to be simpler always to be preferred over those that appear to be more complex? Currently, developmental psychobiological investigation reveals that development is complex and often nonintuitive. Given the assumption of parsimony, then such investigation may appear unnecessarily complicated. Indeed, translation of the developmental psychobiological framework into procedures for practical application would require extensive alteration of nearly all social institutions (Michel \& Moore, 1995). It remains to be determined whether the extra effort needed to conduct developmental psychobiological research is warranted in terms of its eventual practical application.

Current application of developmental neuropsychology is constrained not just by historical precedent, but also by societal values and procedures for reimbursement. Approaches to neuropsychological assessment that begin to incorporate some of the framework characteristic of developmental psychobiology are time consuming and costly (e.g., Kaplan, 1991). For example, Edith Kaplan (1991) proposed that assessment adopt a process approach that would provide information about how the individual performed the tasks of the assessment, rather than information about which aspects of the tasks were and were not completed within the time limits of the test. Theoretically, the information obtained from a process approach specifies exactly the circumstances under which a patient's symptoms disappear and what contextual supports must be provided to enable effective functioning by the brain damaged individual. This information could be used to establish new, nonintuitive, procedures for rehabilitation. That her proposal has not been universally accepted attests to the difficulties facing the emergence of a developmentally rich neuropsychology. That some have adopted her methods attests to the possibility that developmental neuropsychology may be on the verge of achieving a new level of science. Now it is time for developmental psychobiologists to begin specifying the practical applications of their work. Further collaboration between neuropsychologists and psychobiologists should foster such growth.

\section{ACKNOWLEDGMENT}

The research described in this article was supported by Grant 1R0 MH 35528 from the National Institute of Mental Health, Grants 1R01 HD 16107 and HD 22399 from the National Institute of Child Health and Human Development and the DePaul University Research Council, respectively. 


\section{REFERENCES}

Annett, M. (1995). The right shift theory of a genetic balanced polymorphism for cerebral dominance and cognitive processing. Current Psychology of Cognition, 14, 427-480.

Atchley, W. R., \& Hall, B. K. (I99I). A model for development and evolution of complex morphological structures. Biological Reviews, 66, 101-157.

Baldwin, J. M. (I895). The mental development of the child and race. New York: Macmillan. Bateson, P. P. G. (I979). How do sensitive periods arise and what are they for? Animal Behaviour, 27, 470-486.

Bateson, P. P. G. (I98I). Control of sensitivity to the environment during development. In K. Immelmann, G.

W. Barlow, L. Petrinovich, \& M. Main (Eds.), Behavioral development (pp. 432-453). Cambridge, England:

Cambridge University Press.

Bekoff, A. (1988). Embryonic motor output and movement patterns: Relationship to postnatal behavior. In W.

P. Smotherman \& S. R. Robinson (Eds.), Behavior of thefetus (pp. 191-206). Caldwell, NJ: Telford Press.

Blass, E. M. (Ed.). (1986). Developmental psychobiology and developmental neurobiology. New York: Plenum.

Blass, E. M. (Ed.) (I988). Developmentalpsychobiologyand behavioral ecology. New York: Plenum. Bradshaw, J. L., \& Mattingley, J. B. (I995). Clinical neuropsychology: Behavioral and brain science. New York:

Academic.

Bryden, M. P.. \& Steenhuis, R. E. (I99I). Issues in the assessment ofhandedness. In F. L. Kitterle (Ed.), Cerebral laterality: Theory andresearch (pp. 35-51). Hillsdale, NJ: Lawrence Erlbaum Associates, Inc.

Case, R. (I992). The role of the frontal lobes in the regulation of cognitive development. Brain and Cognition, 20, 51-73.

Colombo, J. (1992). The critical period concept: Research, methodology and theoretical issues. Psychological Bulletin, 91, 260-276.

Corballis, M. C. (I995). Evolving theories of handedness. Current Psychology of Cognition, 14, 529-536.

Darian-Smith, C., \& Gilbert, C. D. (I994). Axonal sprouting accompanies functional reorganization in adult cat striate cortex. Nature, 368, 737-740.

Dethier, V. G. (1969). Whose real world? American Zoologist, 9, 241-249.

De Yeo, E. A., Felleman, D. J., Van Essen, D. C., \& McClendon, E. (I994). Multiple processing streams in occipitotemporal cortex. Nature, 371, 151-154.

Efron, R. (1990). The decline andfall ofhemispheric specialization. Hillsdale, NJ: Lawrence Erlbaum

Associates, Inc.

Ellis, A. W. (I987). Intimations of the modularity of mind: Doing cognitive neuropsychology without syndromes. In M. Coltheart, G. Sartori, \& R. Job (Eds.), The cognitive neuropsychology oflanguage (pp. 397408). Hillsdale, NJ: Lawrence Erlbaum Associates, Inc.

Fentress, J. C. (I99I). Analytic ethology and synthetic neuroscience. In P. P. G. Bateson (Ed.), The development and integration ofbehaviour (pp. 77-120). Cambridge, England: Cambridge University Press.

Gesell, A. L. (1946). The ontogenesis of infant behavior. In L. Carmichael (Ed.), Manual of child psychology (pp. 295-331). New York: Wiley.

Gibson, K. R.. \& Peterson, A. C. (Eds.). (I99I). Brain maturation and cognitive development. New York:

Aldine.

Gitterman, M. R. (I999). The critical period: Some thoughts on Grimshaw, etal. (I998). Brain andLanguage, 66, 377-381.

Gottlieb, G. (1992). Individual development and evolution: The genesis of novel behavior. New York: Oxford University Press.

Hogan, J. A., \& Bolhuis, J. J. (Eds.). (I994). Causal mechanisms ofbehavioral development. New York: Cambridge University Press.

Johnson, M. H. (Ed.). (I993). Brain development and cognition: A reader. Oxford, England: Blackwell. Kaplan, E. (I99I). A process approach to neuropsychological assessment. In T. Boll \& B. K. Bryant

(Eds.), Clinical neuropsychology and brain function: Research, measurement, and practice (pp.

81-108). Washington, DC: American Psychological Association.

Kuhn, T. (I962). The structure of scientific revolutions. Chicago: University of Chicago Press. Lehrman, D. S.

(I97I). Behavioral science, engineering, and poetry. In E. Tobach, L. R. Aronson, \& E. 
Shaw (Eds.), The biopsychology of development (pp. 459-471). New York: Academic. Lenneberg, E. H. (I967). The biological foundations of language. New York: Wiley.

LeVay, S. (I99I). A difference in hypothalamic structure between heterosexual and homosexual men. Science, 253, 1034-1037.

Marshall, J. C. (I980). On the biology of language acquisition. In D. Caplan (Ed.), Biological studies of mental processes (pp. 231-245). Cambridge, MA: MIT Press.

McGraw, M. B. (1946). Maturation of behavior. In L. Carmichael (Ed.), Manual of child psychology (pp. 332369). New York: Wiley.

McManus, I. C., \& Bryden, M. P. (I992). The genetics of handedness, cerebral dominance and lateralization. In I. Rapin \& S. J. Segalowitz (Eds.), Handbook of neuropsychology, vol. 6: Child neuropsychology (pp. 115144). Amsterdam: Elsevier.

Merigan, W. H. (I993). Human V4? Current Biology, 3, 226-229.

Merzeni ch, M. M., Recanzone, G. H., Jenkins, W. M., \& Nudo, R. J. (I990). How the brain functionally rewires itself. In M. Arbib \& J. Robinson (Eds.), Natural and artificial parallel computations (pp. 177-210). Cambridge, MA: MIT Press.

Michel, G. F. (I98I). Right-handedness: A consequence of infant supine head-orientation preference? Science, 212, 685-687.

Michel, G. F. (1987). Self-generated experience and the development of lateralized neurobehavioral organization in infants. In J. S. Rosenblatt, C. G. Beer, \& P. J. B. Slater (Eds.), Advances in the study of behavior, vol. 17 (pp. 61-84). New York: Academic.

Michel, G. F. (I99I). Development of infant manual skills: Motorprograms, schemata, or dynamic systems? In J. Fagard \& P. H. Wolff (Eds.), The development oftiming control and temporal organization in coordinated action (pp. 175-199). New York: Elsevier.

Michel, G. F. (I992). Maternal influences on infant hand-use during play with toys. Behavior Genetics, 22, 163176.

Michel, G. F. (I998). A lateral bias in the neuropsychological functioning of human infants. Developmental Neuropsychology, 14, 445-469.

Michel, G. F., \& Harkins, D. A. (I986). Postural and lateral asymmetries in the ontogeny of handedness during infancy. Developmental Psychobiology, 19, 247-258.

Michel, G. F., \& Moore, C. L. (1995). Developmental psychobiology: An interdisciplinary science. Cambridge, MA: MIT Press.

Moore, C. L. (I992). The role ofmaternal stimulation in the development of sexual behavior and its neural basis. Annals of the New York Academy of Sciences, 662, 160-177.

Moore, C. L. (I995). Maternal contributions to mammalian reproductive development and the divergence of males and females. Advances in the Study of Behavior, 24, 47-118.

Moore, C. L., Dou, H., \& Juraska, J. M. (I992). Maternal stimulation affects the number of motor neurons in a sexually dimorphic nucleus of the lumbar spinal cord. Brain Research, 572, 52-56.

Nash, J. (1978). Developmentalpsychology: Apsychobiological approach (2nd ed.). Engelwood Cliffs, NJ: Prentice Hall.

Prechtl, H. F. R. (I982). Regressions and transformations during neurological development. In T. G. Bever (Ed.), Regressions in mental development (pp. 103-118). Hillsdale, NJ: Lawrence Erlbaum Associates, Inc. Previc, F. H. (I99I). A general theory concerning the prenatal origins of cerebral lateralization in humans. Psychological Review, 98, 299-334.

Purves, D., Riddle, D. R., \& LaMantia, A. S. (I992). Iterated patterns of brain circuitry (or how the cortex gets its spots). Trends in Neuroscience, 15, 362-367.

Purves, D., Riddle, D. R., \& LaMantia, A. S. (I993). Reply. Trends in Neuroscience, 16, 180-181. Quartz, S. R., \& Sejnowski, T. J. (I998). The neural basis of cognitive development: A constructivist manifesto.

Behavioral and Brain Sciences, 20, 537-596.

Rovee-Collier, C. (I996). Does early experience affect later behavior? The question of infant memory. In D. H. Ashmead (Organizer), The importance of early experience: A symposium honoring Lewis P. Lipsitt at the 10th Biennial International Conference on Infant Studies, Providence, RI, April 18-21. 
Shallice, T. (1988). From neuropsychology to mental structure. Cambridge, England: Cambridge University Press.

Spinelli, D. N., \& Jensen, F. E. (I982). Plasticity, experience and resource allocation inmotor cortex and hypothalamus. In C. D. Woody (Ed.), Conditioning (pp. 161-169). New York: Plenum.

Spreen, O., Risser, A. H., \& Edgell, D. (I995). Developmental neuropsychology. Oxford, England: Oxford University Press.

Thelen, E., \& Cooke, D. W. (1987). The relation between newborn stepping and later locomotion: A new interpretation. Developmental Medicine and Child Neurology, 29, 380-393.

Thelen, E., \& Smith L. B. (I994). A dynamic systems approach to the development ofcognition and action. Cambridge, MA: MIT Press.

Wolff, P. H., Michel, G. F., Ovrut, M., \& Drake, C. (I990). Rate and timing precision of motor coordination in developmental dyslexia. Developmental Psychology, 26, 349-359.

Yahr, P. (1988). Sexual differentiation ofbehavior in the context of developmental psychobiology. In E. M. Blass (Ed.), Handbook of behavioral neurobiology. Vol. 9. Developmental psychobiology and behavioral ecology (pp. 197-243). New York: Plenum. 